\title{
Journalists as end-users: quality management principles applied to the design process of news automation
}

\section{Laurence Dierickx}

ReSIC, Université Libre de Bruxelles, Belgium.

\begin{abstract}
ISO 9000 refers to a family of standards related to quality management. It defines the concept of quality as the features and characteristics of a product, a process, or a service that bears on its ability to satisfy needs explicitly or implicitly expressed. Standards provide guidance and tools to ensure that products or services will meet users 'requirements. It means that quality must be consistently improved and that risks must be evaluated to be anticipated. The seven principles of the ISO 9000 are here examined through the lenses of a case study conducted within a Belgian newsroom, where a news automation system was developed to support the daily routines of financial journalists. As end-users, they have been involved in the design process. Journalists provided the text templates to automate, based on financial data bought to a German company, while the development of the writing engine was taken in charge by a French start-up. The empirical material was collected through participant observation, including access to all of the working documents. Although it does not guarantee the quality of the content or the end-uses, the standards allowed us to frame a social process where all of the stakeholders were taken into account.
\end{abstract}

Keywords: news automation; professional practices; quality management; standards; ISO 9000. 